\title{
Expression of heat shock protein-coding genes associated with anhydrobiosis in an African chironomid Polypedilum vanderplanki
}

\author{
Oleg Gusev • Richard Cornette • Takahiro Kikawada • \\ Takashi Okuda
}

Received: 10 July 2010 /Revised: 11 August 2010/Accepted: 13 August 2010/Published online: 31 August 2010

(C) The Author(s) 2010. This article is published with open access at Springerlink.com

\begin{abstract}
In order to survive in extreme environments, organisms need to develop special adaptations both on physiological and molecular levels. The sleeping chironomid Polypedilum vanderplanki, inhabiting temporary water pools in semi-arid regions of Africa, is the only insect to have evolutionarily acquired the ability to withstand prolonged complete desiccation at larval stage, entering a state called anhydrobiosis. Even after years in a dry state, larvae are able to revive within a short period of time, completely restoring metabolism. Because of the possible involvement of stress proteins in the preservation of biomolecules during the anhydrobiosis of the sleeping chironomid, we have analyzed the expression of genes encoding six heat shock proteins (Pv-hsp90, Pv-hsp70, Pv-hsc70, Pv-hsp60, Pv-hsp20, and $P v-p 23)$ and one heat shock factor $(P v-h s f 1)$ in dehydrating, rehydrating, and heat-shocked larvae. All examined genes were significantly up-regulated in the larvae upon dehydration and several patterns of expression were detected. Gene transcript of $P v-h s f 1$ was up-regulated within $8 \mathrm{~h}$ of desiccation, followed by large shock proteins expression reaching peak at 24-48 h of desiccation. Heat-shockresponsive $P v$ - $h s p 70$ and $P v$ - $h s p 60$ showed a two-peak expression: in dehydrating and rehydrating larvae. Both small alpha-crystallin heat shock proteins (sHSP) transcripts were accumulated in the desiccated larvae, but showed
\end{abstract}

Electronic supplementary material The online version of this article (doi:10.1007/s12192-010-0223-9) contains supplementary material, which is available to authorized users.

O. Gusev $\cdot$ R. Cornette $\cdot$ T. Kikawada $(\bowtie) \cdot$ T. Okuda $(\bowtie)$

Anhydrobiosis Research Unit,

National Institute of Agrobiological Sciences,

1-2 Ohwashi,

Tsukuba, Ibaraki 305-8634, Japan

e-mail: kikawada@affrc.go.jp

e-mail: oku@affrc.go.jp different expression profiles. Both sHSP-coding genes were found to be heat-inducible, and $P v-h s p 20$ was up-regulated in the larvae at the early stage of desiccation. In contrast, expression of the second transcript, corresponding to $P v-p 23$, was limited to the late stages of desiccation, suggesting possible involvement of this protein in the glass-state formation in anhydrobiotic larvae. We discuss possible roles of proteins encoded by these stress genes during the different stages of anhydrobiosis in P. vanderplanki.

Keywords Anhydrobiosis · Heat shock proteins .

The sleeping chironomid Polypedilum vanderplanki.

Alpha-crystallin proteins $\cdot$ Desiccation stress

\section{Introduction}

Molecular chaperones are a large and diverse group of proteins with property of supporting noncovalent assembly/ disassembly of other macromolecules in the cell (Kregel 2002; Burdon 1986; Frydman 2001). Heat shock proteins (HSP) also actively participate in long-term adaptations to the environmental changes and seasonal developmental patterns in invertebrates. In a number of insect species, upregulation of hsps begins at the start of diapause and decreases back to the normal homeostatic level during the re-initiation of development (Rinehart et al. 2006b, 2007a; Gkouvitsas et al. 2008). During the last decade, involvement of large HSPs (HSP40, HSP60, HSP70, HSP90, and HSP100) in various hypometabolic processes in arthropods has been experimentally confirmed on a number of insect species. In spite of some species-based and HSP-typerelated controversy, it is widely accepted that insect diapause is associated with changes in HSP expression both on transcriptional and translational levels. After an 
initial report in 1998 by Denlinger's group, changes in expression of HSP associated with different types of diapause were confirmed for members of several insect taxa, including Hymenoptera, Coleoptera, Lepidoptera, and Diptera (Benoit et al. 2009; Rinehart et al. 2006b, 2007a; Danks 2000; Gkouvitsas et al. 2008). The growing amount of data suggests that association of HSP with developmental arrest is a common pattern even beyond insects, and temporal changes or continuously higher levels of HSP expression would have impact in developmental progress, thermal resistance, and general level of metabolic activity (Watanabe et al. 2003a). The main functions of HSPs in these examples of development arrest are proposed to be an interaction with other cryoprotectants to increase general stability of the protein pool and a direct action on the suppression of the development (Rinehart et al. 2007a).

Dehydration of cells is one of the most serious stresses, and is crucial for most organisms, since massive irreversible protein-protein aggregation caused by the hydrophobic effect occurs as a compensation for the loss of free water. Such changes in most cases lead to death, as the majority of organisms have limited potency to withstand water loss (Alpert 2006; Goyal et al. 2005; Sakurai et al. 2008a; Bohnert 2000). At the same time, there are examples of anhydrobiosis - the phenomena of maintaining viability for a long period of time under the absence of free water. The best-characterized examples include microorganisms, plants, rotifers, nematodes, tardigrades, crustaceans, and insects (Alpert 2006; Goyal et al. 2004; Watanabe 2006). In such organisms, all biochemical reactions and metabolism are undetectable in the dried state, but anhydrobiotes are able to revive back to the active life in a short period of time after appearance of water. In some groups of organisms, anhydrobiosis is an obligate part of the life cycle, while others continuously maintain the potential to reversibly enter the dry state (Crowe and Madin 1974; Guidetti and Jonsson 2002; Watanabe 2006; Watanabe et al. 2005; Clegg 2001, 2005). While physiological and morphological aspects of anhydrobiosis are relatively well-described, the molecular mechanisms allowing such natural dry preservation of cell organelles and macromolecules are yet to be understood in detail. HSPs have, for a long time, been thought to have high impact on the intracellular processes associated with desiccation tolerance in higher eukaryotes, but only a few members of this group of chaperones have been actually analyzed with a special focus to anhydrobiosis. Two small HSPs, p26 and artemin, have been found in high amounts $(10-15 \%$ of total non-yolk proteins) in the encysted embryos of several branchiopod crustaceans, and a growing amount of data suggests that chaperone activity of these proteins is a key factor for the formation of dry cysts, viable and resistant to environmental stresses (Clegg 2001, 2005; Willsie and Clegg 2001). Accumulation of small HSPs has also been observed in plant seeds (Hoekstra et al. 2001; Kalemba and Pukacka 2008; Wehmeyer and Vierling 2000). Larger HSPs have had even less attention. Recently, Schill and co-authors have demonstrated that at least some isoforms of HSP70-coding genes are up-regulated when tardigrades enter anhydrobiosis and revive back to active metabolism, while other chaperones show no clear pattern of involvement in the process of anhydrobiosis (Schill et al. 2004, 2009; Reuner et al. 2009).

In the present study, we have focused on the African chironomid Polypedilum vanderplanki-the largest known anhydrobiotic animal. The larvae of this chironomid can withstand complete desiccation and maintain viability for years in a dry state, indicating the existence of a highly effective mechanism of long-term preservation of proteins in the dried larvae. The activity of HSPs, together with other protectants (trehalose and LEA proteins) for cells and organelles, has previously been suggested to be of significance for the larvae, enabling protection of the metabolic machinery upon anhydrobiosis (Kikawada et al. 2006, 2007; Nakahara et al. 2008; Watanabe 2006). We have conducted comparative analyses of structural and expression of genes coding six members of main HSP families and a heat shock factor (HSF) in relation to anhydrobiosis of $P$. vanderplanki.

\section{Materials and methods}

\section{Insect rearing}

P. vanderplanki larvae were reared on milk agar under controlled light (13 h light:11 h dark) at 27 to $28^{\circ} \mathrm{C}$. The procedure of desiccation to induce anhydrobiosis is as previously described (Watanabe et al. 2003b), i.e., the larvae were placed on filter paper with $0.44 \mathrm{ml}$ of distilled water in a glass Petri dish (diameter $65 \mathrm{~mm}$, height $20 \mathrm{~mm}$ ), which was set in a desiccator $(20 \times 20 \times 20 \mathrm{~cm})$ with $1 \mathrm{~kg}$ of silica gel. Larvae for RNA and protein expression analyses were sampled according to the time (in hours) passed from the beginning of desiccation (D) and of rehydration (R), correspondingly.

Heat shock treatment

To examine the heat shock response of HSP-coding gene expression, 100 wet active larvae were kept at $42^{\circ} \mathrm{C}$ for $60 \mathrm{~min}$ in a $50-\mathrm{ml}$ tube with preheated deionized water and then transferred to a tube of the same volume of the water at 
$25^{\circ} \mathrm{C}$ during $90 \mathrm{~min}$ for recovery. After that, total RNA was extracted from the larvae for further analysis. The control larvae were kept at $25^{\circ} \mathrm{C}$ continuously in deionized water until RNA extraction.

Chaperones cDNA cloning from P. vanderplanki

In a P. vanderplanki EST database (Kikawada et al. 2006), the clones showing structural similarities to known hsps were isolated, and the corresponding full-length cDNAs were obtained by $5^{\prime}$ - and $3^{\prime}$-RACE using a SMART RACE cDNA amplification kit (Clontech, Mountain View, CA, USA) with specific primers (sequences are available upon request). The full length of $P v$-hsps and $P v-h s f 1$ corresponding cDNAs were subcloned into $\mathrm{pCR}^{\circledR} 4$ TOPO $^{\circledR}$ vector (Invitrogen, Carlsbad, CA, USA) and further used as templates for real-time PCR.

\section{Quantitative real-time PCR}

Total RNA from hydrated, dehydrating, rehydrated, and heat-shocked larvae was extracted using Trizol (Invitrogen) and the RNeasy Mini Kit (Qiagen, Hilden, Germany) and reverse transcribed using Ready-To-Go ${ }^{\mathrm{TM}} \mathrm{T}$ Prime First-Strand Kit (GE Healthcare Bio-Sciences, Piscataway, NJ, USA). The RNA samples from dehydrating and rehydrating larvae were named " $D$ " and " $R$ ", respectively, and numbers correspond to the hours of treatment. Real-time PCR was performed using a LightCycler $^{\circledR} 2.0$ real-time PCR apparatus (Roche Diagnostics, Basel, Switzerland) with SYBR ${ }^{\circledR}$ Green PCR Master Mix (TaKaRa, Ohtsu, Japan).

Amplifications were performed using $1 \times$ SYBR Green PCR mix (TaKaRa) and 10 pmol of each primer. PvEf1-alpha gene (AB490338.1) was used as an internal standard for data normalization and quantification. The expression of each gene was tested in triplicate in each of the three biologically independent experiments. The cycling conditions were as follows: 3 min activation at $95^{\circ} \mathrm{C}, 45$ cycles of $10 \mathrm{~s}$ at $95^{\circ} \mathrm{C}$, $20 \mathrm{~s}$ at $60^{\circ} \mathrm{C}$, and $25 \mathrm{~s}$ at $72^{\circ} \mathrm{C}$. Melting curves from 60 to $99^{\circ} \mathrm{C}$, rising by $1^{\circ} \mathrm{C}$ at each step and pausing $5 \mathrm{~s}$ after each step, and the accompanying software were used for qPCR data normalization and quantification. The full list of primers is described in Supplementary Table S1.

\section{Statistical analysis}

Results of gene expression are reported as means $\pm 95 \%$ CIA (95\% confidence interval) with statistical evaluation performed using a two-tailed Student $t$ test. A difference at $P<0.05$ was considered significant in Prism version 5 (GraphPad Software, San Diego, CA, USA). The full list of reference sequences used in this study is described in Supplementary Table S2.

\section{Results}

Heat shock factor $(P v-h s f 1)$ gene expression in relation to anhydrobiosis

The full sequence for $P v$-hsfl (HM589528) was obtained by a combination of sequence data from the $P$. vanderplanki EST database and RACE cloning. $P v-h s f 1$ has a $15-\mathrm{bp}$ 5'-UTR, a 571-amino acid coding ORF, and a 771-bp 3'-UTR (Supplemental Fig. S1). The deduced amino acid sequence of $P v$ - $h s f 1$ shows the typical characteristics of the HSF 1 family (Wu 1995), including the DNA binding domain and oligomerization domains (Supplemental Fig. S1). Phylogenetical analysis confirmed the identity of Pv-HSF1 (Fig. 1c).

$P v$ - $h s f 1$ expression was examined throughout dehydration and rehydration of the larvae using real-time PCR technique. While transcripts were detected in all samples, relative $P v$-hsfl expression increased in the larvae already in D-8 (corresponding to $8 \mathrm{~h}$ of desiccation) and maintained a high level, up to nearly complete dehydration of the larvae (Fig. 1b). In the desiccated, rehydrating, and completely revived larvae (R 1-48), Pv-hsfl expression was detected at low level as observed in non-stressed active larvae. The $P v$-hsfl gene was also heat shock-inducible (Fig. 1a).

Heat shock protein $90-\mathrm{kDa}(P v-h s p 90)$ gene expression in relation to anhydrobiosis

The full-length sequence for $P v$ - $h s p 90$ (HM589529) was obtained through combination of the sequence from the EST database and RACE cloning of $P$. vanderplanki cDNA. A conceptual translation of the composite cDNA revealed that the ORF encodes a 714-amino-acid protein with classical ATPase domain and HSP90 catalytic domain (Supplemental Fig. S2). The deduced amino acid sequence of $P v-h s p 90$ is structurally similar to cytoplasmic HSP90s and, according to the modern nomenclature, should be classified as a member of HSP90a group (Fig. 1f).

The presence of mRNA encoding Pv-HSP90 was linked to the entering to anhydrobiosis and further rehydration periods. As shown in Fig. 1e, over-expression of $P v$ - $h s p 90$ was detected after $8 \mathrm{~h}$ of desiccation. After approximately $48 \mathrm{~h}$ of desiccation, its expression reached the maximum level, but in completely dried larvae (R0) and during the rehydration mRNAs gradually declined to the level of average metabolic expression pattern at $48 \mathrm{~h}$ after rehydra- 
A

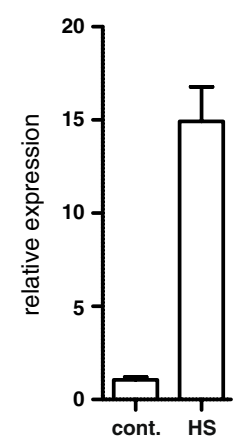

B

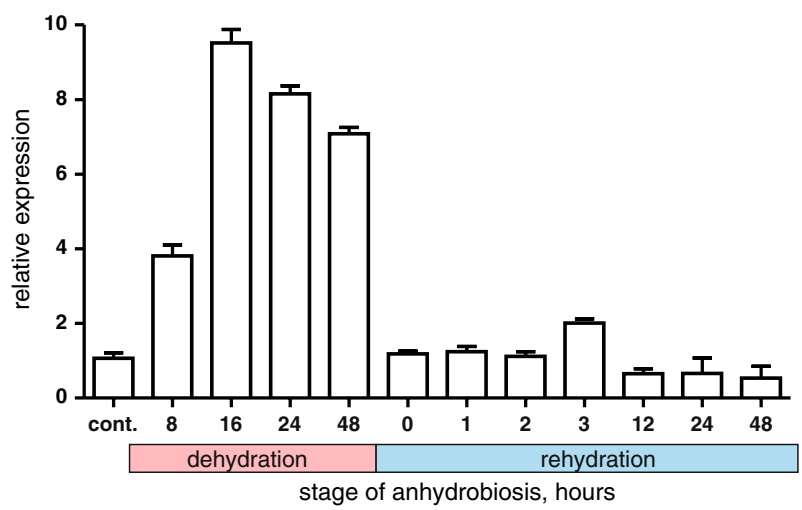

E

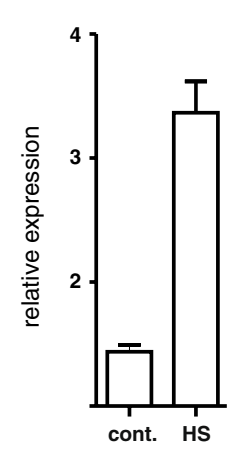

PvHSF-1

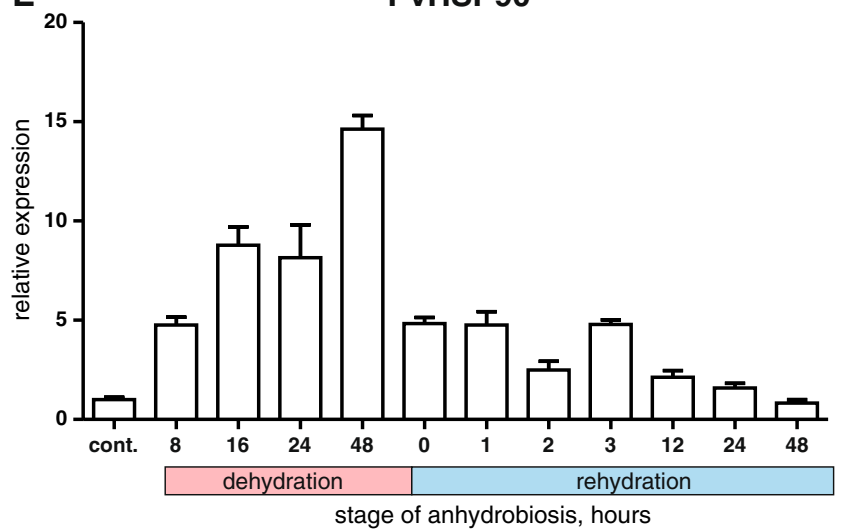

C

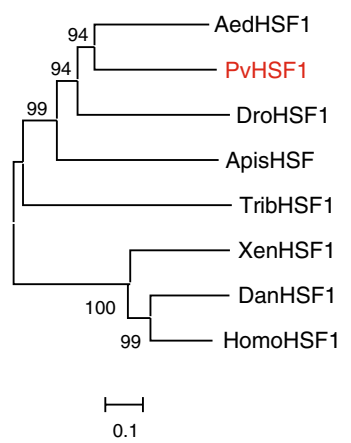

$\mathbf{F}$

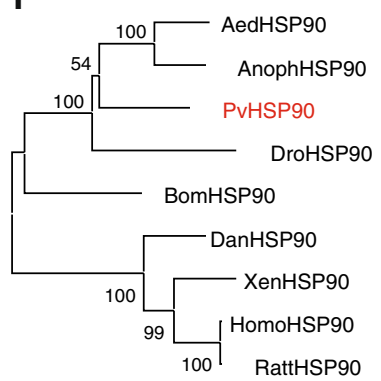

$\longmapsto 0$
Fig. 1 Relative mRNA expression profiles for $P v$ - $h s f 1$ and $P v-h s p 90$ in heat-shocked (a, d) and anhydrobiotic (b, e) chironomid larvae. Values for the mRNA level of each gene were corrected with PvEf1alpha expression level. The level of expression was calculated for each gene relative to the expression in control hydrated larvae (value= 1). Error bars represent mean value $\pm 95 \%$ CI for three replicates. cont. control hydrated larvae. c, e Neighbor-joining tree of the Pv-HSF1 and Pv-HSP90 amino acid sequences tion. The expression of $P v-h s p 90$ was also enhanced by heat shock (Fig. 1d).

Expression of genes coding two $70-\mathrm{kDa}$ heat shock proteins $(P v-h s p 70$ and $P v-h s c 70)$ in relation to anhydrobiosis

We have cloned two full transcripts corresponding to proteins from HSP70 family (Fig. 2). The first transcript, named $P v-h s c 70$ (HM589530), is a full-length clone of 2,272 bp, with a 1,962-bp ORF encoding a 654-aa protein. The deduced amino acid sequence contained the typical HSP70 protein family signatures 1-3 (Supplemental Fig. S3) and showed the highest similarity to Chironomus HSC70 (AAN14525) (Fig. 2c). No up-regulation of $P v$ hsc70 was observed under heat stress, and we defined the transcript as a cognate 70-kDa HSP (Fig. 2a). Analysis of $P v$ - $h s c 70$ expression showed that up-regulation of the mRNA was first detectable in the larvae subjected to 8-h drying and kept increasing with maximum level occurring at $48 \mathrm{~h}$ of dehydration. The mRNA level showed a lower value in completely dried and just rehydrated larvae and finally dropped to the average nonstress level comparable to the 12-24 h revived larvae (Fig. 2b).

The second full-length transcript, $P v-h s p 70$ (HM589531), consisted of 2,441 bp with a 1,974-bp ORF, encoding 658-aa protein. The deduced amino acid sequence, with the highest similarity to Aedes hsc70 (ABF18258), showed the presence of all conservative HSP70 family structural signatures (Fig. 2f, Supplemental Fig. S4). Over-expression of the transcript in response to heat stress was observed, and we defined the gene as inducible HSP70 (Fig. 2, Supplemental Fig. S4). Though up-regulation of $P v-h s p 70$ was already detectable in larvae after $8 \mathrm{~h}$ of desiccation (Fig. 2e) and reaching at its peak in the larvae subjected to 48-h drying, the level of expression was lower than that of $P v-h s c 70$ (Fig. 2b). Furthermore, a second peak of up-regulation appeared in the larvae $3 \mathrm{~h}$ after the beginning of rehydration. The expression returned to the average metastatic level at the stage of $24 \mathrm{~h}$ of rehydration (R-24, Fig. 2e). 
A

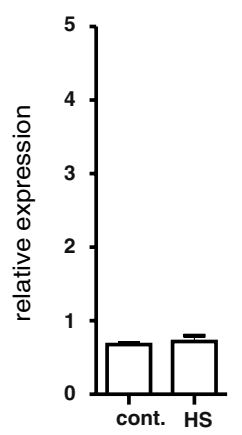

D

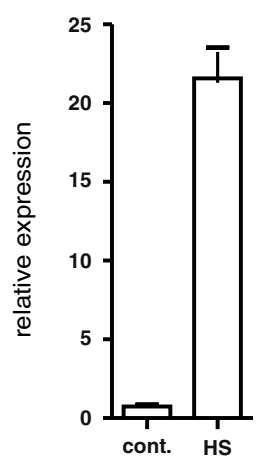

G

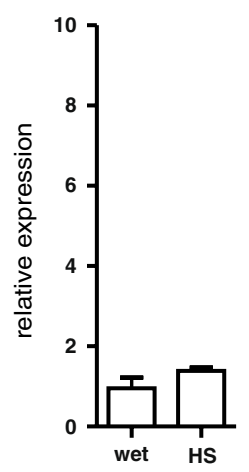

B

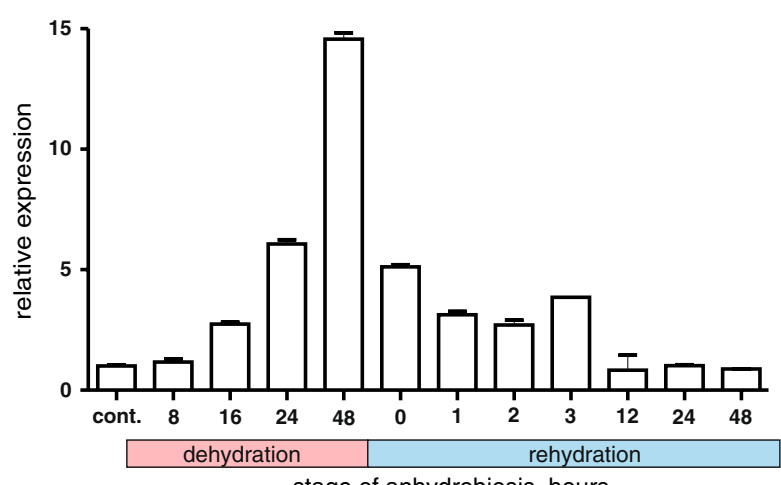

stage of anhydrobiosis, hours

E

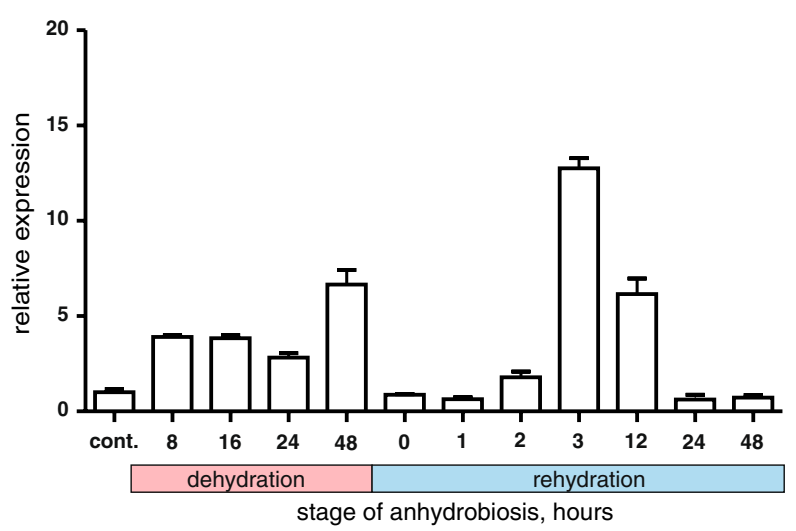

H

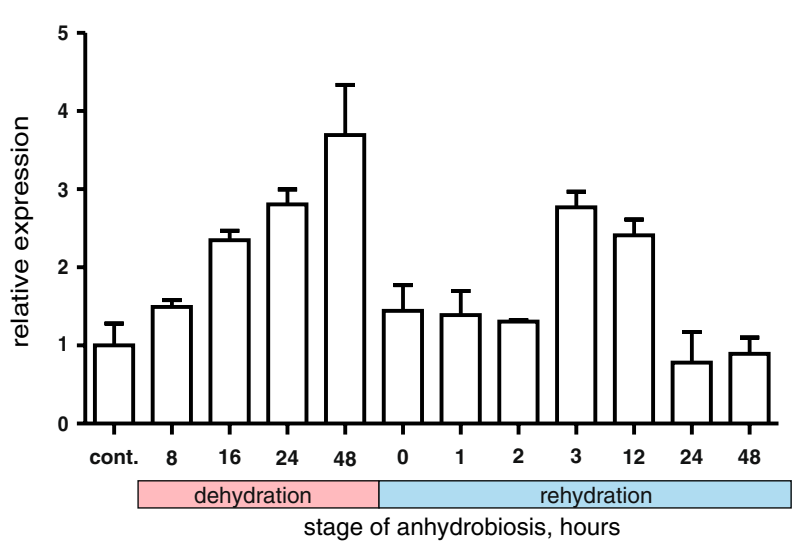

C

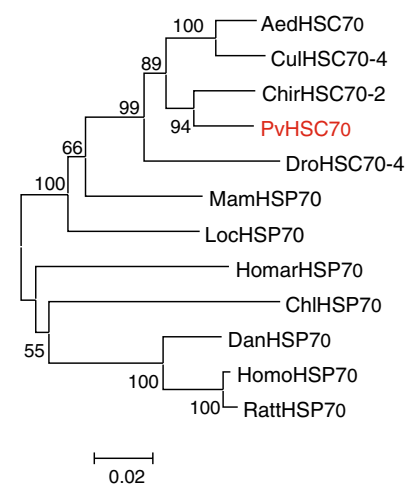

$\mathbf{F}$

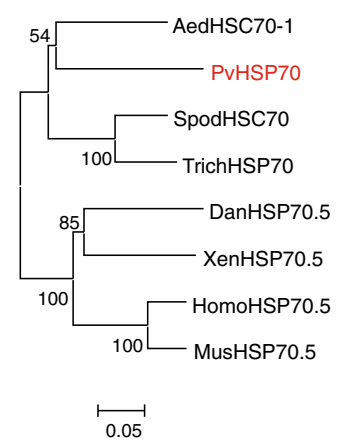

I

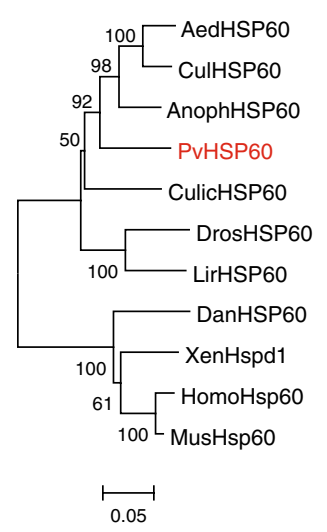

Fig. 2 Relative mRNA expression profiles for $P v$ - $h s p 70, P v$ - $h s c 70$, and $P v$-hsp60 in anhydrobiotic $(\mathbf{b}, \mathbf{e}, \mathbf{h})$ and heat-shocked $(\mathbf{a}, \mathbf{d}, \mathbf{g})$ larvae. Values for the mRNA level of each gene were corrected for expression level of PvEf1-alpha, and the relative level of expression

Heat shock protein $60-\mathrm{kDa}(P v-h s p 60)$ gene expression during anhydrobiosis

The cDNA encoding the $P$. vanderplanki HSP60 (Pv-hsp60, HM589532) was 2,243 bp in length (Supplemental Fig. S5) with a 1,710-bp ORF. The deduced protein comprised 569 amino acids. The first 26 triplets downstream of the changes for each gene was calculated using that of control hydrated larvae as standard (value $=1$ ). Error bars represent mean value $\pm 95 \%$ CI for three replicates. cont. control hydrated larvae. c, f, i Neighborjoining tree of Pv-HSC70 and Pv-HSP60 amino acid sequences

initiation codon ATG encode amino acids that together have the necessary characteristics of a mitochondrial presequence. A GGM motif was located at the carboxyl terminus; other domains indicating typical mitochondrial HSP60 (GroEL_like type I chaperonin) properties were found in the deduced protein sequence (Supplemental Fig. S5, Fig. 2i). The highest-scoring amino acid similar- 
ities were to HSP60 (chaperonins) from Anopheles (XP 318461.2), Culicoides (AAB94640.1), and Aedes (XP_001661764). The gene was not heat-inducible (Fig. 2g). Expression activity of $P v-h s p 60$ in the larvae was similar to that of $P v$ - $h s p 70$ : a two-peak pattern during entering and reviving from anhydrobiosis. We observed initial increase of the expression already in the larvae subjected to $8 \mathrm{~h}$ of desiccation and some further increase (2-4 folds) up to the nearly complete desiccated state of the larvae (D-48). At the same time, level of expression of $P v$ hsp60 in rehydrating larvae was initially on the lower level compared with that in the dehydrating larvae, but showed the second peak in a few hours after complete revival of the larvae (Fig. 2h).

Expression of genes coding two small chaperones $p v-h s p 20$ and pv-p23 (p26 homolog) during anhydrobiosis

The last two analyzed chaperones belong to the group of low molecular mass proteins containing alpha-crystallin domain (alpha-HSP). One of the small HSP-coding genes $(P v-h s p 20$, HM589533) is a full-length clone of $835 \mathrm{bp}$ with a 522-bp ORF (Supplemental Fig. S6), coding a putative $20-\mathrm{kDa}$ protein $(173 \mathrm{aa})$ with highest similarity to Culex HSP22 (XP_001847195) and Drosophila HSP27 (ABX80641.1) (Fig. 3c).

The second small HSP ( $P v-p 23$, HM589534) is represented by full-length clone of $885 \mathrm{bp}$, with a 588 -bp ORF (Supplemental Fig. S7), coding a putative 22.8-kDa protein (196 aa) that most closely resembles Belgica sHSP (ABF01017) and Artemia p26 (ABC41139) (Fig. 3f). Both HSPs showed presence of the conservative alpha-crystallin domain, assuming formation of large oligomeric complexes as a structural prerequisite for the chaperone activity. At the same time, the domain showed a low level of crosssimilarities (less than $35 \%$ on the amino acid level), suggesting that activity patterns and cellular functions of the proteins would be different. In addition, translated sequence of $P v-h s p 20$ showed the presence of a typical WDPF motif (amino acids residues 10-30, Supplemental Fig. S6 and S7), whereas $P v-p 23$ did not.

We found that both $P v$-hsp20 and $P v-p 23$ were upregulated in the larvae subjected to heat shock (Fig. 3a, d). Furthermore, both genes showed over-expression associated with anhydrobiotic processes in the larvae, but their expression patterns were different. Under normal nonstressed conditions, the expression of $P v-h s p 20$ was at the low level (Fig. 3b, e) and then up-regulated already after $8 \mathrm{~h}$ of desiccation and showed the highest level of expression in the nearly dry and just revived larvae and followed by slow declining to the normal, non-stressed level at $48 \mathrm{~h}$ of rehydration. In contrast, expression of $P v$ p23 was dehydration-inducible and restricted to the late stages of dehydration of the larvae showing its maximum level in the completely desiccated and just revived larvae and dropped to non-stress lowest level as early as $12 \mathrm{~h}$ after rehydration (Fig. 3e).

\section{Discussion}

In this study, we have demonstrated that expression of genes coding several members of the main HSP groups is tightly linked to the anhydrobiosis of $P$. vanderplanki larvae. Figure 4 summarizes the periods of up-regulation of HSP-corresponding genes in chironomid larvae during the dehydration-rehydration cycle.

\section{Large HSP-encoding genes}

Experimental data related to involvement of large heat shock proteins specifically in anhydrobiosis in invertebrates is limited to several papers related to $h s p$ expression in tardigrades, where it is shown that there are at least three hsp70-like genes (all heat shock up-regulated) with different expression profiles in relation to anhydrobiosis. All three isoforms show significant up-regulation after revival of the tardigrades to active stage (Schill et al. 2004; Jonsson and Schill 2007; Reuner et al. 2009). At the same time, after further analysis of other groups of $h s p$, the authors concluded that chaperones are not involved in anhydrobiosis in water bears.

In the present study, we have isolated two different isoforms of $h s p 70$ and they both are significantly upregulated during entire process of anhydrobiosis in the chironomid larvae (Fig. 2b, e). Pv-hsp 70, a heat-shockresponsive isoform, referred to gene encoding as real HSP70, showed an initial up-regulation pattern at the beginning of dehydration of the larvae and later, an even higher peak, at the stage corresponding to $3 \mathrm{~h}$ after rehydration (Fig. 2e). Such patterns of inducible $h s p 70$ expression are similar to that in tardigrades, where it was suggested that chaperone over-expression was related to refolding of the proteins damaged during formation of the "tun" and revival back to active life after anhydrobiosis (Schill et al. 2004). Pv-hsc70, a cognate isoform, instead shows only one-peak up-regulation, with a gradual increase in the expression level during desiccation, reaching a maximum level at the final step of dry larvae formation (stage D-48). Then, continuous decrease of mRNA level was observed during rehydration, approaching average nonstressed level at the stage R12 (Fig. 2b). Based on absolute copy numbers estimated by real-time PCR and EST database (data not shown) and on the expression pattern, $P v-h s c 70$ seems to be the dominant HSP70 isoform both in wet (active) and dry (anhydrobiotic) larvae. Activity of 
A

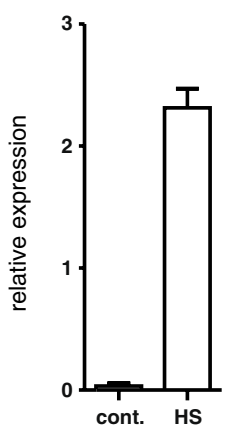

D

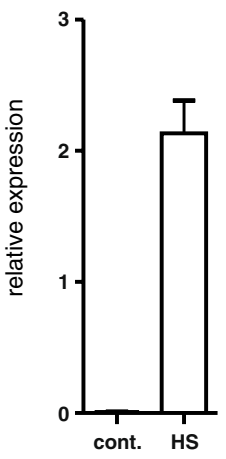

B

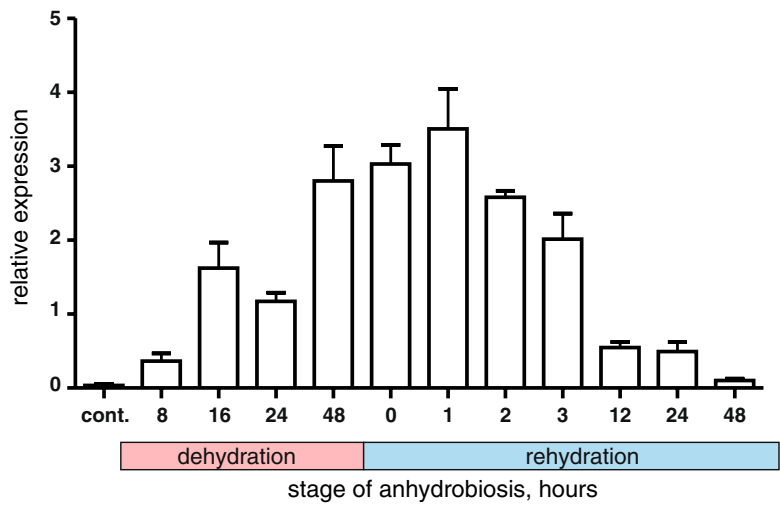

E

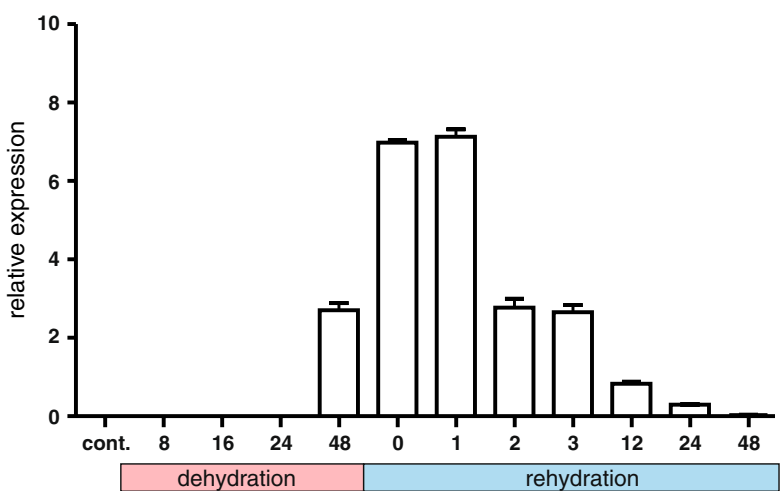

stage of anhydrobiosis, hours

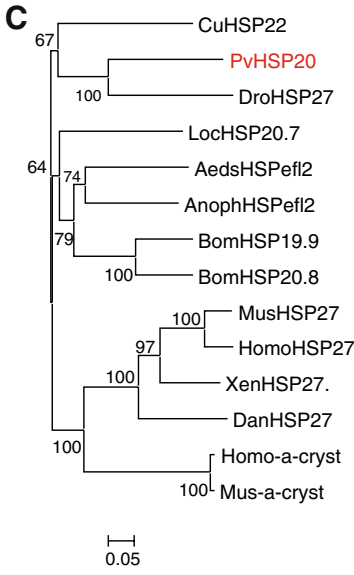

$\mathbf{F}$

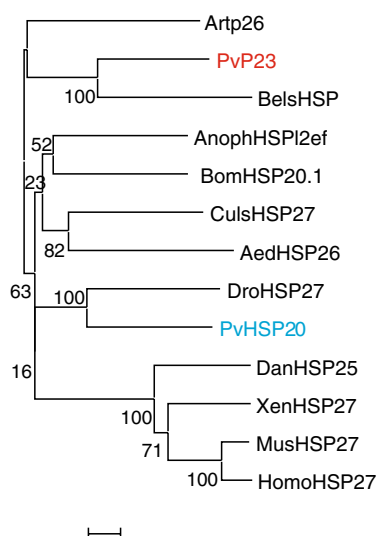

$\stackrel{\longmapsto}{0.05}$
Fig. 3 Relative mRNA expression profiles for $P v-h s p 20$ and $P v-p 23$ in anhydrobiotic (b, e) and heat-shocked (a, d) larvae. Values for the mRNA level of each gene were corrected for expression level of EF1$a l p h a$, and the relative level of expression changes for each gene was calculated using that of control hydrated larvae as standard (value $=1$ ). Error bars represent mean value $\pm 95 \%$ CI for three replicates. cont. control hydrated larvae. c, f Neighbor-joining tree of the Pv-HSP20 and $\mathrm{Pv}-\mathrm{p} 23$ amino acid sequences

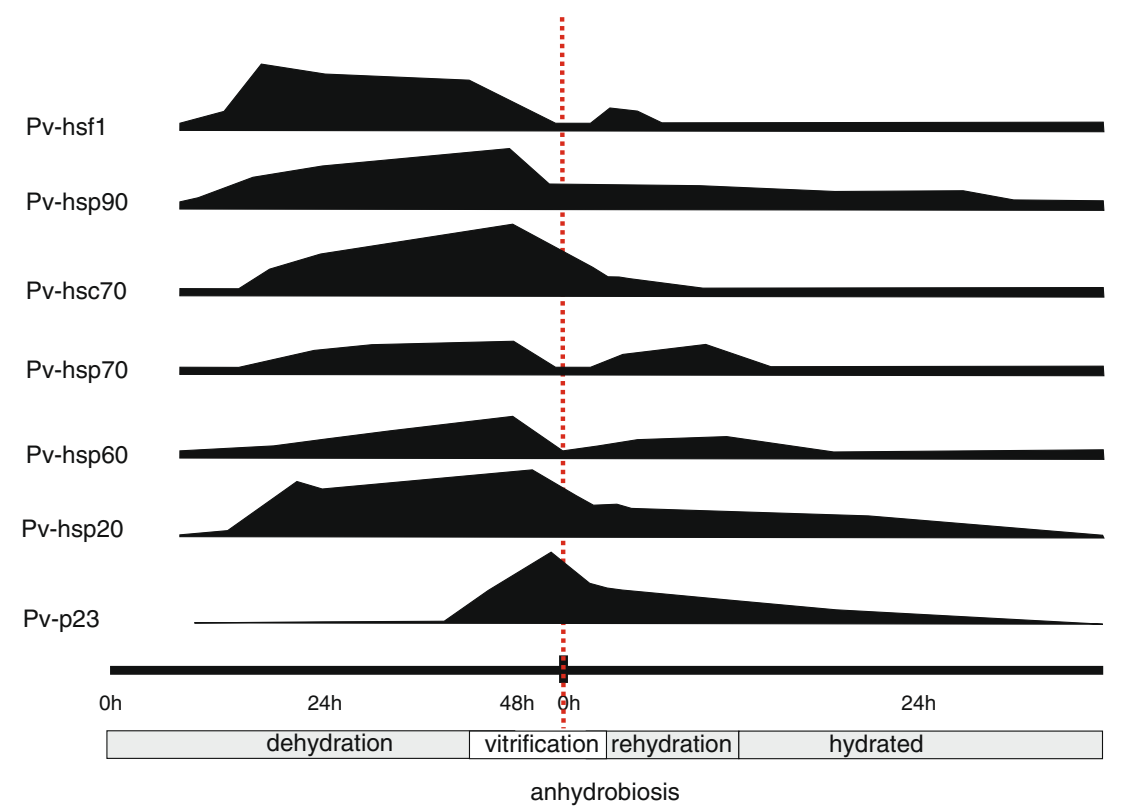

Fig. 4 Temporal representations of anhydrobiosis stages, in which genes encoding heat shock proteins are up-regulated in the larvae of the sleeping chironomid 
HSC70 (cognate form of HSP70) is usually tightly linked to de novo protein synthesis machinery and during hypometabolic processes, such as insect diapause or under stress conditions (including desiccation), where the expression of hsc70 is reported to be unchanged (Denlinger 2002; Sonoda et al. 2006a) or even down-regulated (Gkouvitsas et al. 2009). No specific studies on HSC70 in anhydrobiotic invertebrates have been reported so far, but our results suggest that protein product of $P v-h s c 70$ is an active participant of anhydrobiotic processes in the larvae. While in the cases of typical hypometabolic processes both transcription and translation activity drop to lower level, in the larvae of $P$. vanderplanki de novo synthesis of several groups of LEA proteins and conversion of glycogen to trehalose followed by its delivery to cells are critical factors defining the success of anhydrobiosis (Kikawada et al. 2006, 2008; Watanabe et al. 2007). As water leaves cells during dehydration, the synthesis of LEA proteins and other molecular factors essential for the anhydrobiotic state of larvae have to be completed under conditions of increased intracellular concentration of macromolecules. The overexpression of $P v-h s c 70$ probably reflects an active participation of $\mathrm{Pv}-\mathrm{HSC} 70$ in this synthesis process.

Members of HSP90 family are considered to be the among the most abundant chaperones in the cytoplasm of eukaryotic cells, but, to the best of our knowledge, no studies focusing on the relation of these chaperones to anhydrobiotic processes have been reported to date (Goyal et al. 2005; Schill et al. 2009; Watanabe 2006). In spite of the fact that HSP90 is important for multiple developmental and metabolic networks, information about its involvement in insect diapause remains controversial. Depending on the type of diapause and species, the expression level of the chaperone decreases, increases, or remains unchanged (Lopez-Martinez and Denlinger 2008; Rinehart et al. 2007a; Sonoda et al. 2006b). Recently, it was found that expressions of both HSC70 and HSP90 in the larvae of flesh fly were unresponsive to dehydration (Hayward et al. 2004; Rinehart et al. 2006a, 2007a). In our study, up-regulation of $P v$ - $h s p 90$ gene in the larvae of $P$. vanderplanki was observed throughout the entire process of entering into, and revival from, anhydrobiosis with a dynamic of expression similar to that of $P v$-hsc70 (Figs. 1 and 2). The similar patterns of over-expression for both genes continued during rehydration of the larvae, assuming the reinitiation of productive protein folding pathways, in which both HSC70 and HSP90 play a fundamental role (Leung and Hightower 1997; Young et al. 2001). This might suggest the co-activity of both chaperones as a sign of maturation of the proteins involved in anhydrobiosis process (Rajapandi et al. 2000).

$P v$ - $h p$ 60 also was the only gene coding a HSP60 family member found in $P$. vanderplanki EST database and analyzed in this study (our unpublished data). In contrast to that of other chaperones, the mRNA expression of $P v$ hsp60 showed a much lower degree of change during entire anhydrobiosis (Fig. 2h). Another member of the HSP60 family, TCP-1, involved in the folding of cytoskeleton proteins was recently found to be tightly linked with diapause and low-temperature survival in insects and brine shrimp (Rinehart et al. 2007a; Wang et al. 2007), but at present we have no data about the activity of the homologous gene in the larvae of P. vanderplanki.

\section{Genes encoding small (crystallin-like) HSPs}

Here we have cloned and analyzed expression of two genes coding small heat shock proteins $(P v-h s p 20$ and $P v-p 23$; Fig. 3). Both proteins possess crystallin domains but show differences in primary structure and expression pattern during anhydrobiosis.

Pv-HSP20 has all the typical structural features of the sHSP family and is phylogenetically close to HSP27 of Drosophila. Expression of this gene is also up-regulated by heat shock (Fig. 3). The expressional pattern of $P v$ - $h s p 20$ resembles those of small HSPs appearing in developing plant seeds ahead of the processes associated with desiccation (Coca et al. 1994; Wehmeyer et al. 1996). To date, there have been no studies focusing on the participation of HSP27 homologs in the anhydrobiosis of invertebrates. At the same time, activity of this gene is tightly linked to the resistance to environmental changes and to metabolic suppression during developing and diapausing stages of insects and other invertebrates (Rinehart et al. 2007b; Hayward et al. 2004; Saravanakumar et al. 2008). Up-regulation of $P v$-hsp 20 was already observed in the larvae $8 \mathrm{~h}$ after the start of desiccation, and a high level of the corresponding mRNA was maintained for at least $24 \mathrm{~h}$ after revival from anhydrobiosis. We assume that $P v-h s p 20$ encodes a member of sHSPs and that its initial upregulation in dehydrating larvae may be related to chaperone activity in the larvae suffering from different factors associated with water deficit, including molecular crowding and oxidative stress. The dynamic of $P v$-hsp20 mRNA abundance is similar to that of $P v-h s p 90$ and $P v-h s f 1$, but at the present step we have no data on joint activity of these chaperones in the larvae (Fig. 4).

In contrast, expression of the second small HSPcoding gene, $P v-p 23$, was undetectable in wet and semidry larvae (Fig. 3e). Based on phylogenetic analysis, we consider Pv-P23 to be a desiccation-inducible ortholog of Artemia's p26-a small chaperone functionally involved in the process of anhydrobiosis in anoxic cysts of lower crustaceans (Willsie and Clegg 2001). In Artemia's cysts, p26 increases in concentration, occupying $10-15 \%$ of total non-yolk protein biomass, and migrates to the nucleus in dehydrating cysts. Artemia's p26 has been proposed to act 
as a chaperone, stabilizing nuclear acids (Clegg 2005; Liang and MacRae 1999; Willsie and Clegg 2001).

Drastic increase of $P v-p 23$ mRNA in the larvae occurred between stages D-24 and D-48, i.e., during the period preceding vitrification of the cells and tissues. Such a dehydration-specific activity of the Pv-p23-coding gene might suggest a direct link between this protein and anhydrobiosis. As a member of the crystallin family, Pvp23 would also form an oligomeric structure and possibly be involved in the vitrification of larval tissues on the late stages of desiccation (Sakurai et al. 2008b). In summary, we have shown that the initiation of anhydrobiosis-related processes in $P$. vanderplanki is associated with up-regulated expression of the genes coding major groups of HSP. We suggest that HSPs constitute an important part of anhydrobiosis-related changes in the cells of $P$. vanderplanki larvae. At the same time, while our discussion is based on the assumption that mRNA expression reflects the activity of mature HSPs, it should be noted that a temporal shift between gene expression and the activity of mature stress proteins was observed in some cases (Kostal and TollarovaBorovanska 2009). Consequently, more data are needed to make a conclusion on the activity of HSPs in anhydrobiotic larvae. Thus, further studies with a special focus on cellular and tissue-specific localization, defining the role of each member of this family, including careful analysis of protein expression of each isoform, will need to be carried out for a better understanding of the origin and machinery of long-term water-free preservation of biomolecules during anhydrobiosis in the sleeping chironomid.

Open Access This article is distributed under the terms of the Creative Commons Attribution Noncommercial License which permits any noncommercial use, distribution, and reproduction in any medium, provided the original author(s) and source are credited.

Acknowledgment We thank Peter Wilson for his help and advice during the course of paper preparation. This study was supported in part by the Promotion of Basic Research Activities for Innovative Bioscience (PROBRAIN), and by KAKENHI, a Grant-in-Aid for Young Scientists (A).

\section{References}

Alpert P (2006) Constraints of tolerance: why are desiccation-tolerant organisms so small or rare? J Exp Biol 209(9):1575-1584

Benoit JB, Lopez-Martinez G, Elnitsky MA, Lee RE, Denlinger DL (2009) Dehydration-induced cross tolerance of Belgica antarctica larvae to cold and heat is facilitated by trehalose accumulation. Comp Biochem Physiol A 152(4):518-523

Bohnert HJ (2000) What makes desiccation tolerable? Genome Biol 1 (2):REVIEWS1010

Burdon RH (1986) Heat-shock and the heat-shock proteins. Biochem J 240(2):313-324
Clegg JS (2001) Cryptobiosis - a peculiar state of biological organization. Comp Biochem Physiol B 128(4):613-624

Clegg JS (2005) Desiccation tolerance in encysted embryos of the animal extremophile, Artemia. Integr Comp Biol 45(5):715-724

Coca MA, Almoguera C, Jordano J (1994) Expression of sunflower low-molecular-weight heat-shock proteins during embryogenesis and persistence after germination-localization and possible functional implications. Plant Mol Biol 25(3):479-492

Crowe JH, Madin KA (1974) Anhydrobiosis in tardigrades and nematodes. T Am Microsc Soc 93(4):513-524

Danks HV (2000) Dehydration in dormant insects. J Insect Physiol 46 (6):837-852

Denlinger DL (2002) Regulation of diapause. Annu Rev Entomol 47:93-122

Frydman J (2001) Folding of newly translated proteins in vivo: the role of molecular chaperones. Annu Rev Biochem 70:603-647

Gkouvitsas T, Kontogiannatos D, Kourti A (2008) Differential expression of two small Hsps during diapause in the corn stalk borer Sesamia nonagrioides (Lef.). J Insect Physiol 54(12):1503-1510

Gkouvitsas T, Kontogiannatos D, Kourti A (2009) Cognate hsp70 gene is induced during deep larval diapause in the moth Sesamia nonagrioides. Insect Mol Biol 18(2):253-264

Goyal K, Browne JA, Walton LJ, Pinelli C, Rastogi RK, Burnell AM, Tunnacliffe A (2004) Molecular anhydrobiology: identifying molecules implicated in invertebrate anhydrobiosis. Integr Comp Biol 44(6):560-560

Goyal K, Walton LJ, Browne JA, Burnell AM, Tunnacliffe A (2005) Molecular anhydrobiology: identifying molecules implicated in invertebrate anhydrobiosis. Integr Comp Biol 45(5):702-709

Guidetti R, Jonsson KI (2002) Long-term anhydrobiotic survival in semi-terrestrial micrometazoans. J Zool 257:181-187

Hayward SAL, Rinehart JP, Denlinger DL (2004) Desiccation and rehydration elicit distinct heat shock protein transcript responses in flesh fly pupae. J Exp Biol 207(6):963-971

Hoekstra FA, Golovina EA, Buitink J (2001) Mechanisms of plant desiccation tolerance. Trends Plant Sci 6(9):431-438

Jonsson KI, Schill RO (2007) Induction of hsp70 by desiccation, ionising radiation and heat-shock in the eutardigrade Richtersius coronifer. Comp Biochem Physiol B Biochem Mol Biol 146 (4):456-460

Kalemba EM, Pukacka S (2008) Changes in late embryogenesis abundant proteins and a small heat shock protein during storage of beech (Fagus sylvatica L.) seeds. Environ Exp Bot 63(1-3):274-280

Kikawada T, Nakahara Y, Kanamori Y, Iwata K-i, Watanabe M, McGee B, Tunnacliffe A, Okuda T (2006) Dehydration-induced expression of LEA proteins in an anhydrobiotic chironomid. Biochem Biophys Res Commun 348(1):56-61. doi:10.1016/j.bbrc.2006.07.003

Kikawada T, Saito A, Kanamori Y, Nakahara Y, Iwata KI, Tanaka D, Watanabe M, Okuda T (2007) Trehalose transporter 1, a facilitated and high-capacity trehalose transporter, allows exogenous trehalose uptake into cells. Proc Natl Acad Sci U S A 104 (28): $11585-11590$

Kikawada T, Saito A, Kanamori Y, Fujita M, Snigorska K, Watanabe M, Okuda T (2008) Dehydration-inducible changes in expression of two aquaporins in the sleeping chironomid, Polypedilum vanderplanki. Biochim Biophys Acta 1778(2):514-520

Kostal V, Tollarova-Borovanska M (2009) The $70 \mathrm{kDa}$ heat shock protein assists during the repair of chilling injury in the insect, Pyrrhocoris apterus. Plos One 4(2):e4546

Kregel KC (2002) Heat shock proteins: modifying factors in physiological stress responses and acquired thermotolerance. J Appl Physiol 92(5):2177-2186

Leung SM, Hightower LE (1997) A 16-kda protein functions as a new regulatory protein for hsc70 molecular chaperone and is identified as a member of the nm23/nucleoside diphosphate kinase family. J Biol Chem 272(5):2607-2614 
Liang P, MacRae TH (1999) The synthesis of a small heat shock/ alpha-crystallin protein in Artemia and its relationship to stress tolerance during development. Dev Biol 207(2):445-456

Lopez-Martinez G, Denlinger DL (2008) Regulation of heat shock proteins in the apple maggot Rhagoletis pomonella during hot summer days and overwintering diapause. Physiol Entomol 33 (4):346-352

Nakahara Y, Watanabe M, Fujita A, Kanamori Y, Tanaka D, Iwata K, Furuki T, Sakurai M, Kikawada T, Okuda T (2008) Effects of dehydration rate on physiological responses and survival after rehydration in larvae of the anhydrobiotic chironomid. J Insect Physiol 54(8):1220-1225

Rajapandi T, Greene LE, Eisenberg E (2000) The molecular chaperones hsp90 and hsc70 are both necessary and sufficient to activate hormone binding by glucocorticoid receptor. J Biol Chem 275(29):22597-22604

Reuner A, Hengherr S, Mali B, Forster F, Arndt D, Reinhardt R, Dandekar T, Frohme M, Brummer F, Schill RO (2009) Stress response in tardigrades: differential gene expression of molecular chaperones. Cell Stress Chaperones 15(4):423-430

Rinehart JP, Hayward SAL, Elnitsky MA, Sandro LH, Lee RE, Denlinger DL (2006a) Continuous up-regulation of heat shock proteins in larvae, but not adults, of a polar insect. Proc Natl Acad Sci U S A 103(38):14223-14227

Rinehart JP, Robich RM, Denlinger DL (2006b) Enhanced cold and desiccation tolerance in diapausing adults of Culex pipiens, and a role for hsp70 in response to cold shock but not as a component of the diapause program. J Med Entomol 43(4):713-722

Rinehart JP, Li A, Yocum GD, Robich RM, Hayward SA, Denlinger DL (2007) Up-regulation of heat shock proteins is essential for cold survival during insect diapause. Proc Natl Acad Sci U S A 104(27):11130-11137

Sakurai M, Furuki T, Akao K, Tanaka D, Nakahara Y, Kikawada T, Watanabe M, Okuda T (2008) Vitrification is essential for anhydrobiosis in an African chironomid, Polypedilum vanderplanki. Proc Natl Acad Sci U S A 105(13):5093-5098

Saravanakumar R, Ponnuvel KM, Qadri SMH (2008) Expression of metabolic enzyme genes and heat-shock protein genes during embryonic development in diapause and non-diapause egg of multivoltine silkworm Bombyx mori. Biologia 63(5):737-744

Schill RO, Steinbruck GH, Kohler HR (2004) Stress gene (hsp70) sequences and quantitative expression in Milnesium tardigradum (Tardigrada) during active and cryptobiotic stages. J Exp Biol 207(Pt 10):1607-1613

Schill RO, Mali B, Dandekar T, Schnolzer M, Reuter D, Frohme M (2009) Molecular mechanisms of tolerance in tardigrades: new perspectives for preservation and stabilization of biological material. Biotechnol Adv 27(4):348-352

Sonoda S, Ashfaq M, Tsumuki H (2006a) Cloning and nucleotide sequencing of three heat shock protein genes (hsp90, hsc70, and hsp 19.5) from the diamondback moth, Plutella xylostella (L.) and their expression in relation to developmental stage and temperature. Arch Insect Biochem Physiol 62(2):80-90

Sonoda S, Fukumoto K, Izumi Y, Yoshida H, Tsumuki H (2006b) Cloning of heat shock protein genes (hsp90 and hsc70) and their expression during larval diapause and cold tolerance acquisition in the rice stem borer, Chilo suppressalis Walker. Arch Insect Biochem Physiol 63(1):36-47

Wang WW, Meng B, Chen WH, Ge XM, Liu S, Yu J (2007) A proteomic study on postdiapaused embryonic development of brine shrimp (Artemia franciscana). Proteomics 7(19):35803591

Watanabe M (2006) Anhydrobiosis in invertebrates. Appl Entomol Zool 41(1):15-31

Watanabe M, Kikawada T, Okuda T (2003) Increase of internal ion concentration triggers trehalose synthesis associated with cryptobiosis in larvae of Polypedilum vanderplanki. J Exp Biol 206(Pt 13):2281-2286

Watanabe M, Kikawada T, Fujita A, Okuda T (2005) Induction of anhydrobiosis in fat body tissue from an insect. J Insect Physiol 51(6):727-731

Watanabe M, Nakahara Y, Sakashita T, Kikawada T, Fujita A, Hamada N, Horikawa DD, Wada S, Kobayashi Y, Okuda T (2007) Physiological changes leading to anhydrobiosis improve radiation tolerance in Polypedilum vanderplanki larvae. J Insect Physiol 53(6):573-579

Wehmeyer N, Vierling E (2000) The expression of small heat shock proteins in seeds responds to discrete developmental signals and suggests a general protective role in desiccation tolerance. Plant Physiol 122(4):1099-1108

Wehmeyer N, Hernandez LD, Finkelstein RR, Vierling E (1996) Synthesis of small heat-shock proteins is part of the developmental program of late seed maturation. Plant Physiol 112 (2): 747-757

Willsie JK, Clegg JS (2001) Nuclear p26, a small heat shock/ alpha-crystallin protein, and its relationship to stress resistance in Artemia franciscana embryos. J Exp Biol 204(13):23392350

Wu C (1995) Heat shock transcription factors: structure and regulation. Annu Rev Cell Dev Biol 11:441-469

Young JC, Moarefi I, Hartl FU (2001) Hsp90: a specialized but essential protein-folding tool. J Cell Biol 154(2):267-273 